\title{
Placental Hofbauer cells assemble and sequester HIV-1 in tetraspanin and DC SIGN positive compartments that are accessible to neutralizing antibodies
}

\author{
Erica L Johnson ${ }^{1,2^{*}}$, Hin Chu ${ }^{1,2}$, Siddappa N Byrareddy ${ }^{3}$, Paul Spearman ${ }^{1,2}$, Rana Chakraborty ${ }^{1,2}$ \\ From 2nd International Science Symposium on HIV and Infectious Diseases (HIV SCIENCE 2014) \\ Chennai, India. 30 January - 1 February 2014
}

\section{Background}

Within monocyte derived macrophages, HIV-1 accumulates in virus containing compartments (VCCs) that are largely inaccessible to the external environment, which implicate these cells as HIV-1 reservoirs. During mother to child transmission, placental macrophages (Hofbauer Cells [HCs]) are viral targets, and have shown to be infected in vivo and sustain low levels of viral replication in vitro, however, the risk of in utero transmission is less than $7 \%$. The role of these primary macrophages as viral reservoirs is largely undefined.

\section{Methods}

With consent, term placentas from 20 HIV-1 seronegative women were obtained following caesarian section. VCCs were evaluated with 3D confocal microscopy and correlated with electron microscopy. Co localization $\mathrm{R}$ values (Pearson's correlation) were quantified with co localization module of Volocity5.2.1. Replication kinetics following siRNA and neutralization studies were evaluated using p24 ELISA.

\section{Results}

We demonstrate primary $\mathrm{HCs}$ assemble and sequester HIV-1 in VCCs with localization specific to intracellular vesicles and the plasma membrane. These compartments are enriched in endosomal/lysosomal markers, including CD9, CD81, CD63 and LAMP 1, along with DC SIGN. Following internalization, we observed HIV-1 accumulation in acidified compartments. Remarkably, these compartments are accessible via the cell surface and can be targeted by exogenously applied small molecules and HIV-1 specific neutralizing antibodies (NAbs). In addition, broadly NAbs (4E10 and VRC01) neutralized HIV-1 infected HCs and involve the participation of Fc $\gamma \mathrm{RI}$ for inhibition.

\section{Conclusion}

These findings suggest placental HCs possess intrinsic adaptations facilitating sequestration of HIV-1, and may serve as a protective viral reservoir to permit viral degradation, neutralization and antiretroviral drug entry in utero.

\section{Authors' details}

'Department of Pediatrics; Emory University; Atlanta, Georgia, 30322, USA ${ }^{2}$ Children's Center for Immunology and Vaccines; Children's Healthcare of Atlanta, USA. ${ }^{3}$ Department of Pathology \& Laboratory Medicine, Emory University School of Medicine, \& Emory Vaccine Center, Atlanta, Georgia, 30322, USA.

Published: 27 May 2014

* Correspondence: eljohns@emory.edu

${ }^{1}$ Department of Pediatrics; Emory University; Atlanta, Georgia, 30322, USA

Full list of author information is available at the end of the article

doi:10.1186/1471-2334-14-S3-O5

Cite this article as: Johnson et al:: Placental Hofbauer cells assemble and sequester HIV-1 in tetraspanin and DC SIGN positive compartments that are accessible to neutralizing antibodies. BMC Infectious Diseases 201414 (Suppl 3):05. 